\title{
Foot Trajectory Generation of Hydraulic Quadruped Robots on Uneven Terrain
}

\author{
HyoungKwon Kim*, Daehee Won*, Ohung Kwon*, Tae-Ju Kim*, Sang-Seok Kim*, and Sangdeok Park* \\ * Division for Applied Robot Technology, Korea Institute of Industrial Technology, \\ Ansan, Korea, (Tel:+82-31-8040-6293; e-mail:\{clew21, daehee, ohung210,re94, hirock, sdpark\}@kitech.re.kr)
}

\begin{abstract}
This paper proposes trot pattern generation and online control methods for a legged robot to carry heavy-loads and move fast on the uneven terrain. The trot pattern is generated from the frequency modulated pattern generation method based on the frequency modulated oscillator in order for the legged robots to operate outdoors with the static and dynamic mobility. The efficiency and performance of the proposed are verified through the computer simulation and experiments using qRT-1/-2. In experiments, qRT which is a 2-legged and 2-wheeled robot and a front drive system vehicle with hydraulic linear actuators is used. The robot has trotted gaits at speeds up to $1.3 \mathrm{~m} / \mathrm{s}$ on the even surface, walked up and down the 20 degree inclines, and walked at $0.7 \mathrm{~m} / \mathrm{s}$ on the uneven surface. Also it has carried over $100 \mathrm{~kg}$ of the total weights including over $40 \mathrm{~kg}$ of the payload.
\end{abstract}

\section{INTRODUCTION}

There is an increasing need for robots to have the versatile abilities, that is, the fast movement and transportation power. Many researchers have studied multi-legged robots because they have more versatility than wheeled-robots and more stability than one-leg and biped robots which have the limits to carry heavy loads fast because of joint actuators with low power [1-8]. In general, in order to increase the walking speed and transportation power of a legged vehicle and improve dynamic stability, the robots requires joint actuators with the large bandwidth and the high output power, and the robot control system which manages the dynamics of robot's behavior to keep it balanced, steer, navigate, and regulate its energy as conditions vary.

Recently, control methods for the quadruped robots using the pneumatic and hydraulic actuators were proposed [9-10]. Guihard et al. developed a hybrid robot composed of wheels and legs with the pneumatic actuators [9]. However, it is difficult to control due to the high nonlinearity characteristics of pneumatic system. Playter et al. developed a quadruped robot that walks, runs, and climbs on the rough terrain and carries heavy loads [10]. The quadruped robot is powered by a gasoline engine that drives a hydraulic actuation system, and the legs are articulated like an animal's, and have the compliant elements that absorb shock and recycle the energy from one step to the next.

This paper is related to a trot pattern generation and online control method for a legged robot to carry heavy-loads and move fast on the uneven terrain. The efficiency of each method is verified through the computer simulation and the experiment using a legged robot, qRT-1/-2. qRT-1 is a front drive system vehicle with the hydraulic linear actuators and can carry heavy-loads on back legs with a wheel. It is about $1.0 \mathrm{~m}$ high, $1.0 \mathrm{~m}$ long and $0.5 \mathrm{~m}$ wide, and weights about $60.0 \mathrm{~kg}$, except the power pack. It has an on-board computer

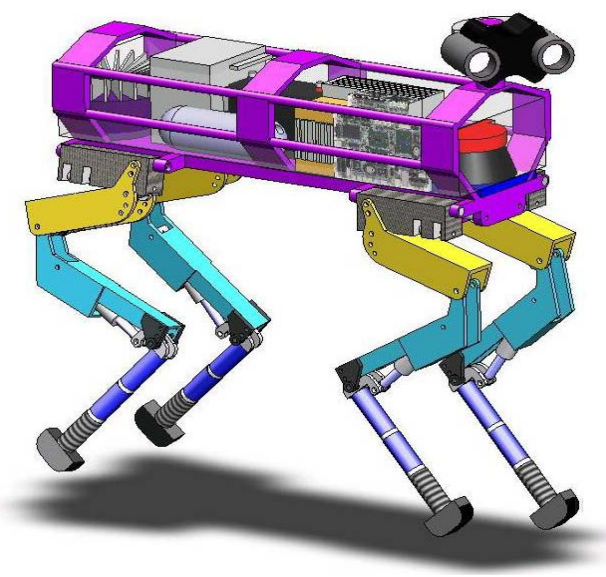

Fig. 1 A quadruped robot, qRT-1

that controls the locomotion, servos the legs and handles a wide variety of sensors. It has demonstrated the trotting gaits at speeds up to $1.3 \mathrm{~m} / \mathrm{s}$ on the even surface, walked up and down the 20 degree inclines, and walked at $0.7 \mathrm{~m} / \mathrm{s}$ on the uneven surface. Also it has carried over $100 \mathrm{~kg}$ of the total weights including over $40 \mathrm{~kg}$ of the payload.

\section{TRAJECTORY GENERATION}

\subsection{Kinematic Model for the Trajectory Generation}

There are a lot of locomotion patterns for the legged robots, such as walking, pacing, trotting, galloping, and so on. As the most available locomotion pattern, the trot pattern is generated so that the legged robots move even though one wants any desired speed in limited range. Figures 1 and 2 show coordinates and parameters of the quadruped robot, qRT-1. First, legs of the simplified quadruped model are tied 


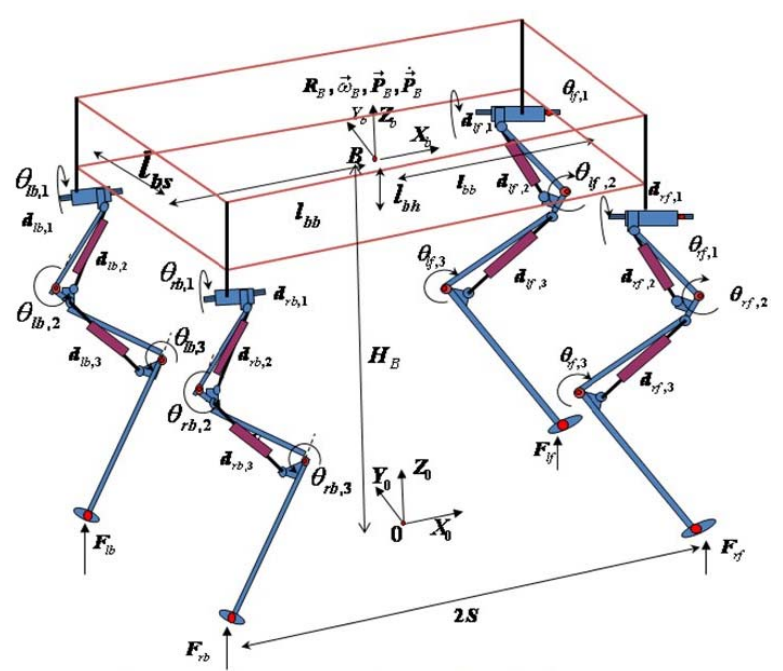

Fig.2 Coordinates and parameters of a quadruped robot, qRT-1

so as to behave like one leg[11], as shown in Fig. 3(a). That is called a virtual biped model which is composed of a body and two legs, as shown in Fig. 3(b). The locomotion trajectory which is specified at the body and the swing foot is defined about the virtual biped model. And then, the trajectory of the virtual biped model is transformed into the quadruped robot model, as shown in Fig. 3(c).

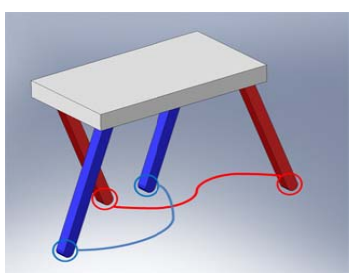

(a) Simplified quadruped robot

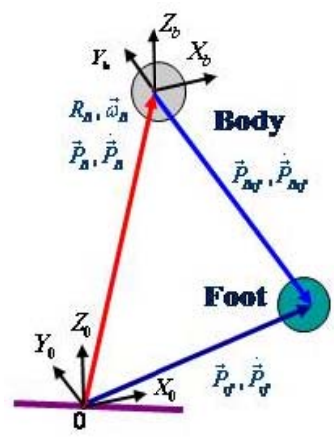

(b) Virtual biped model

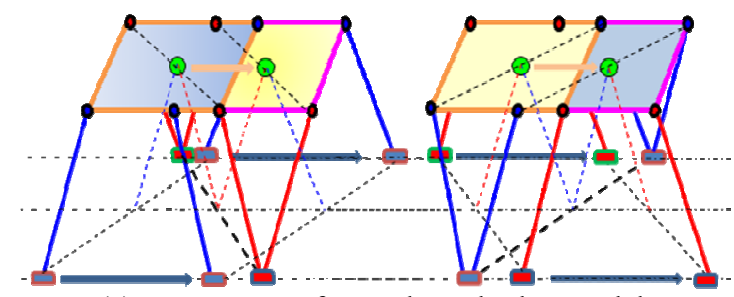

(c) Trot pattern of a quadruped robot model

Fig.3 Trajectory generation for the trotting
In Fig. 3(b), the position of a swing foot about a supporting foot is described as

$$
\overrightarrow{\boldsymbol{P}}_{f}=\overrightarrow{\boldsymbol{P}}_{B}+\boldsymbol{R}_{B} \overrightarrow{\boldsymbol{P}}_{B f},
$$

where $\overrightarrow{\boldsymbol{P}}_{\boldsymbol{f}}$ and $\overrightarrow{\boldsymbol{P}}_{\boldsymbol{B}}$ are the position vector of a swing foot and the position vector of a body frame about the fixed frame on the ground, respectively. And $\boldsymbol{R}_{\boldsymbol{B}}$ and $\overrightarrow{\boldsymbol{P}}_{\boldsymbol{B} \boldsymbol{f}}$ are the orientation matrix of a body frame and the position vector of a swing foot about the body frame. The time derivative of the position vector of a swing foot in Eq. (1) is obtained as

$$
\begin{aligned}
\dot{\overrightarrow{\boldsymbol{P}}}_{f} & =\dot{\overrightarrow{\boldsymbol{P}}}_{B}+\vec{\omega}_{B} \times \boldsymbol{R}_{B} \overrightarrow{\boldsymbol{P}}_{B f}+\boldsymbol{R}_{B} \dot{\overrightarrow{\boldsymbol{P}}}_{B f} \\
& =\dot{\overrightarrow{\boldsymbol{P}}}_{B}+\vec{\omega}_{B} \times \boldsymbol{R}_{B} \overrightarrow{\boldsymbol{P}}_{B f}+\boldsymbol{R}_{B} \boldsymbol{J} \dot{\vec{\theta}}_{f},
\end{aligned}
$$

where $\dot{\boldsymbol{R}}_{\boldsymbol{B}}=\vec{\omega}_{\boldsymbol{B}} \times \boldsymbol{R}_{\boldsymbol{B}}$ and $\dot{\overrightarrow{\boldsymbol{P}}}_{\boldsymbol{B} f}=\boldsymbol{J} \dot{\vec{\theta}}_{f}$. And $\dot{\vec{\theta}}_{f}$ and $\boldsymbol{J}$ are the joint angular velocity and the Jacobian matrix, respectively. Therefore, the joint angular velocity is obtained as follows:

$$
\dot{\vec{\theta}}_{f}=\left(\boldsymbol{R}_{B} \boldsymbol{J}\right)^{-1}\left(\dot{\overrightarrow{\boldsymbol{P}}}_{f}-\dot{\overrightarrow{\boldsymbol{P}}}_{B}-\vec{\omega}_{B} \times \boldsymbol{R}_{B} \overrightarrow{\boldsymbol{P}}_{B f}\right),
$$

where $\quad \overrightarrow{\boldsymbol{P}}_{\boldsymbol{B} f}=\boldsymbol{R}_{\boldsymbol{B}}^{-1}\left(\overrightarrow{\boldsymbol{P}}_{f}-\overrightarrow{\boldsymbol{P}}_{\boldsymbol{B}}\right) \cdot \dot{\overrightarrow{\boldsymbol{P}}}_{\boldsymbol{B}}, \overrightarrow{\boldsymbol{P}}_{\boldsymbol{B}}, \vec{\omega}_{\boldsymbol{B}}$ and $\boldsymbol{R}_{\boldsymbol{B}}$ are determined based on an inverted pendulum model under the assumption that the total mass of the robot is concentrated on the center of body. It satisfies the dynamic walking stability. And then $\dot{\overrightarrow{\boldsymbol{P}}}_{f}$ and $\overrightarrow{\boldsymbol{P}}_{f}$ are determined by the frequency modulated pattern generated method given in Section 2.2.

\subsection{Frequency Modulated Pattern Generation Method}

Quadruped robots need to have the abilities to move on the uneven ground. Therefore, in this paper, the new pattern generation method based on the sigmoidal function is proposed. It makes the shape of foot trajectory changed to be easily adjustable according to the terrain condition such as a gravelly field and a slope.

First, a sinusoidal function is defined to generate the periodical oscillating motion. This function is so-called the frequency modulated oscillator (FMO) in this paper.

$$
\begin{aligned}
& \psi_{\text {osc }}(\phi)=\sin (2 \pi f t+\phi), \\
& \dot{\psi}_{\text {osc }}(\phi)=2 \pi f \cdot \cos (2 \pi f t+\phi),
\end{aligned}
$$

where $f$ is the frequency of the oscillator and it is defined as $f=v_{\text {ref }} / S_{\text {ref }}$, where $v_{\text {ref }}$ and $s_{\text {ref }}$ are the desired speed and stride of the robot. These variables are very useful to the pattern generation of the transition region of the gait motion such as the transitions from the pacing to the trotting or from the trotting to the galloping. It is possible that the pattern can generate by the increasing or decreasing of these variables. And $\phi$ is the phase difference between left and right legs according to the gait type, such as a pace, a trot, and a gallop. The horizontal and vertical trajectories of the robot are generated by depending on $f$ and $\phi$.

Second, when the stride, the velocity, the moving direction, and the gait type of the robot is decided, and then the 
horizontal trajectory of the front two feet is defined from Eq. (4) as follows:

$$
\mathbf{P}_{X Y}=\frac{S_{r e f}}{2} \cdot \gamma \cdot \boldsymbol{\mu} \cdot \boldsymbol{\psi} \cdot \theta
$$

where the $\mathbf{P}_{X Y}$ is $\left[\begin{array}{llll}X_{F R} & Y_{F R} & X_{F L} & Y_{F L}\end{array}\right]^{\mathrm{T}}$, it is the foot trajectories of the robot on the $\mathrm{X}-\mathrm{Y}$ plane, the $\psi$ is the frequency modulated oscillation matrix (FMOM), the $\boldsymbol{\mu}$ is the leg activation matrix (LAM).

In Eq. (5), The FMOM( $\boldsymbol{\psi})$ is given by

$$
\boldsymbol{\psi}=\left[\begin{array}{cc}
\psi_{o s c}\left(\phi_{F R}^{H}\right) \cdot \mathbf{I}_{2} & \mathbf{O}_{2} \\
\mathbf{O}_{2} & \psi_{o s c}\left(\phi_{F L}^{H}\right) \cdot \mathbf{I}_{2}
\end{array}\right]
$$

where $\mathbf{I}_{n}$ and $\mathbf{O}_{n}$ are $n$-by- $n$ identity and zero matrix.

FMOM is defined by the phase difference between each leg according to the sequence of the gait, such as a pace, a trot, and a gallop.

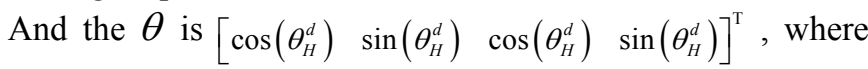
the $\theta_{H}^{d}$ is the desired heading angle.

$$
\gamma=\left\{\begin{array}{cl}
1, & \text { if forward direction } \\
-1, & \text { if backward direction } \\
0, & \text { if hold on }
\end{array}\right\},
$$

where $\gamma$ value is determined according to the desired moving direction of the robot.

Finally, the vertical trajectories of the front two feet are defined as

$$
\mathbf{P}_{Z}=\left[\begin{array}{c}
\frac{Z_{r e f}}{1+\alpha \cdot \exp \left(\beta \cdot \mu_{F R} \cdot \psi_{o s c}\left(\phi_{F R}^{V}\right)\right)} \\
\frac{Z_{r e f}}{1+\alpha \cdot \exp \left(\beta \cdot \mu_{F L} \cdot \psi_{o s c}\left(\phi_{F L}^{V}\right)\right)}
\end{array}\right]+\mathbf{Z}_{i}^{\text {Slope }},
$$

where the $\mathbf{P}_{Z}$ is $\left[\begin{array}{ll}Z_{F R} & Z_{F L}\end{array}\right]^{\mathrm{T}}$, it is the foot trajectories of the robot on the vertical axis, $Z_{\text {ref }}$ is the desired vertical foot position, and $\alpha$ and $\beta$ are parameters to decide of the trajectory shape.

In order to gait, the $\operatorname{LAM}(\boldsymbol{\mu})$ that decides whether the robot moves a leg or not is given by

$$
\boldsymbol{\mu}=\left[\begin{array}{cc}
\mu_{F R} \cdot \mathbf{I}_{2} & \mathbf{O}_{2} \\
\mathbf{O}_{2} & \mu_{F L} \cdot \mathbf{I}_{2}
\end{array}\right]
$$

where $\mu_{i}=\left\{\begin{array}{c}1, \text { if } \psi_{\text {osc }}\left(\phi_{i}^{V}\right)>0 \\ 0, \text { otherwise }\end{array}\right\}, i=F R, F L$, and FR and FL mean the front right and left leg.

When the robot goes up and down the slope or to moves on the uneven surface, the vertical trajectory $\mathbf{P}_{Z}$ is automatically compensated by $\mathbf{Z}_{i}^{\text {Slope }}$ as follows:

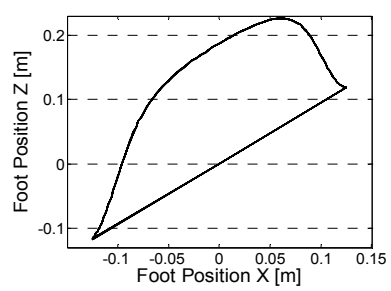

Slope angle: $70^{\circ}$

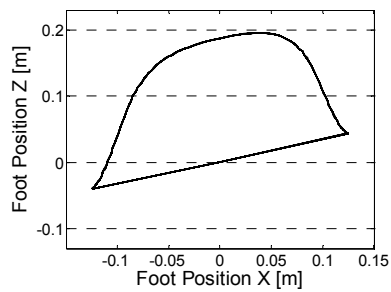

Slope angle: $20^{\circ}$

Fig. 4 Foot position on the slope surface during a step in the sagittal plane.

$$
\mathbf{Z}_{i}^{\text {Slope }}=\left[\begin{array}{c}
X_{F R} \cdot \sin \left(\theta_{F R}^{\text {Slope }}\right) \\
X_{F L} \cdot \sin \left(\theta_{F L}^{\text {Slope }}\right)
\end{array}\right],
$$

where if $\theta_{i}^{\text {Slope }}$, the slope angle of ground under the $i$-th foot, is not zero, $\mathbf{Z}_{i}^{\text {Slope }}$ is activated. Otherwise it is zero and $\mathbf{Z}_{i}^{\text {Slope }}$ is not activated. And then the locomotion trajectory is generated trajectory for the robot to move on the even surface. For example, if the gait type is a trotting, then the phase difference of each leg are $\phi_{F R}^{H}=-\pi / 2, \phi_{F L}^{H}=\pi / 2$, $\phi_{F R}^{V}=\phi_{F R}^{H}+\pi / 2$, and $\phi_{F L}^{V}=\phi_{F L}^{H}+\pi / 2$.

Figure 4 shows the foot trajectory during a step in the sagittal plane when the robot moves on the slope surface. The foot trajectory is changed to be suited for the slope angle. And also Fig. 5 shows the foot trajectories over time on the slope surface. Figure 6 shows the foot trajectory when the robot moves on slope and uneven surfaces. The first obstacle is applied to the front right leg (FR leg), the second obstacle is applied to the front left leg (FL leg), and the third obstacle is applied at both FR and FL leg.
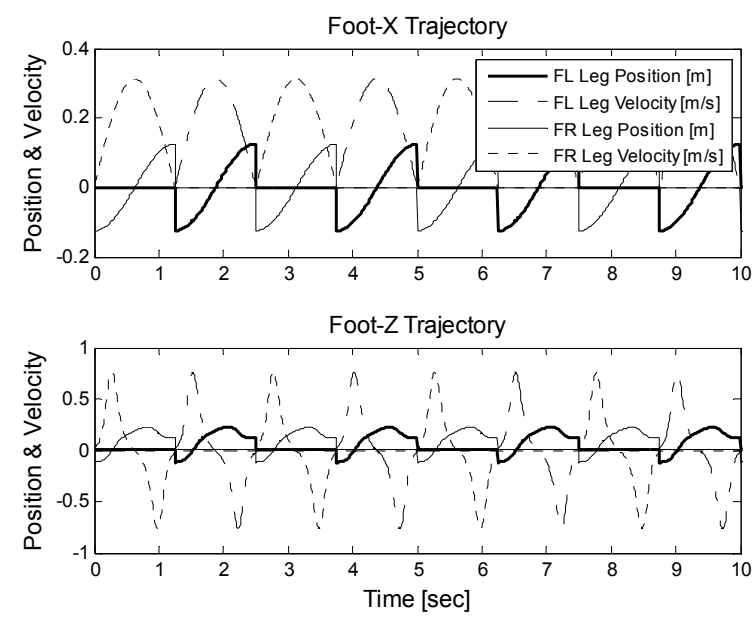

Fig. 5 Foot trajectory to move on the terrain of $45^{\circ}$ slope angle. 


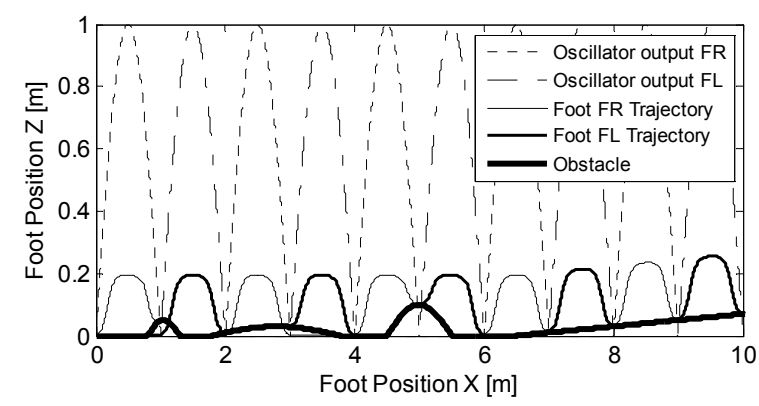

Fig.6 Foot trajectories are changed continuously on the sagittal plane when the quadruped robot moves on the uneven and slope surfaces.
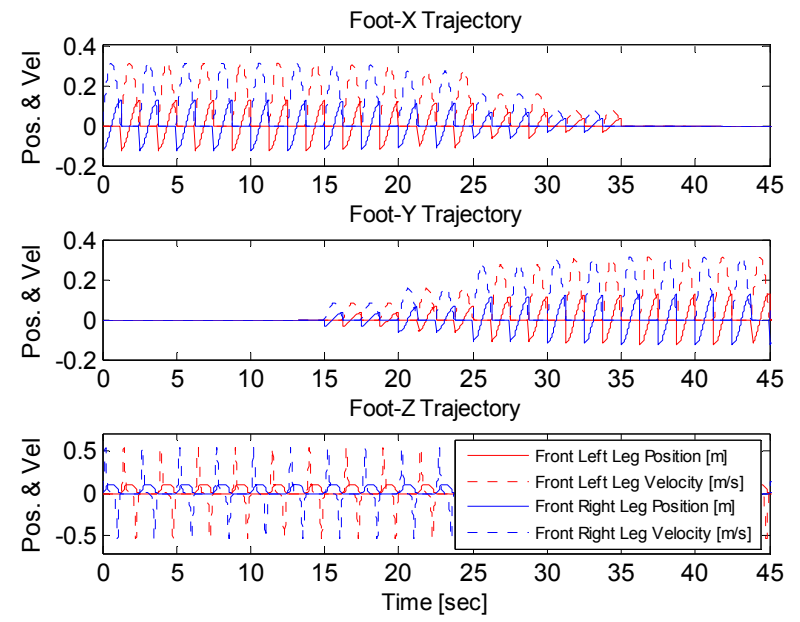

(a) Foot position and velocity on $\mathrm{X}, \mathrm{Y}$, and $\mathrm{Z}$ axes about the time

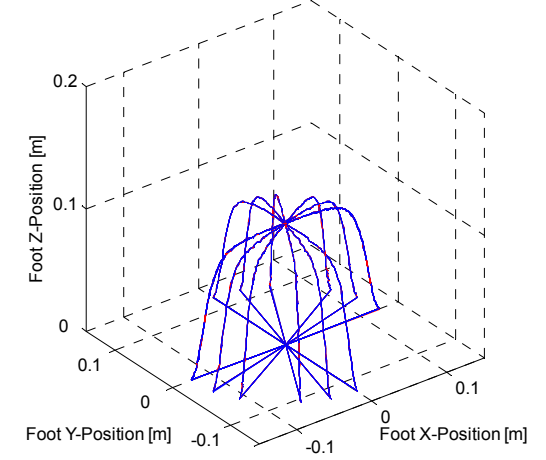

(b) Foot position on $\mathrm{X}-\mathrm{Y}-\mathrm{Z}$ axes according to the heading angle of the robot

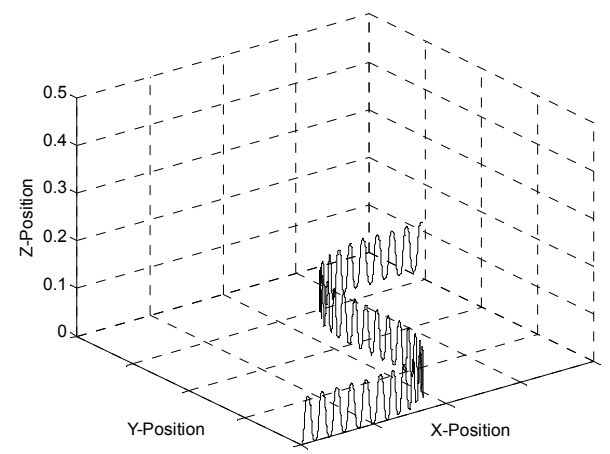

(c) Foot trajectory of the robot on $\mathrm{X}-\mathrm{Y}-\mathrm{Z}$ axes

Fig.7 Foot trajectory according to change the heading angle $\left(0^{\circ} \rightarrow 15^{\circ} \rightarrow 30^{\circ} \rightarrow 60^{\circ} \rightarrow \cdots\right)$
Figure 7 shows the foot trajectories when the quadruped robot moves with a change of direction on the even surface.

Thus, because of a very simple structure, it is possible to the real-time adaption according to the variation of the terrain conditions and directly generate of the various foot trajectories by the change of some parameters, such as $v_{\text {ref }}$, $S_{r e f}, \phi, \alpha$, and $\beta$, and by measure the height of obstacle or the slope angle on the uneven or even surface with the moving direction command.

\section{ON-LINE COMPENSATOR}

Following the commanded trajectory in the free swing motion, the foot comes into contact with the ground. A collision force is generated during the transition phase from the free swing motion to the constrained motion. To avoid large forces, the contact force is modulated by changing the desired vertical position of the contact foot. The environment between the contact force and the foot can be expressed by a spring-damper model. Thus,

$$
\begin{aligned}
\boldsymbol{F}_{e} \boldsymbol{H}_{e} & =\boldsymbol{K}_{d} \boldsymbol{z}_{f c}+\boldsymbol{D}_{d} \dot{z}_{f c} \\
& \approx \boldsymbol{K}_{d} \boldsymbol{z}_{f c, k}+\boldsymbol{D}_{d}\left(\boldsymbol{z}_{f c, k}-\boldsymbol{z}_{f c, k-1}\right) / \Delta \boldsymbol{t} .
\end{aligned}
$$

Hence we have

$$
z_{f c, k}=\frac{F_{e}+H_{B} D_{d} z_{f c, k-1} / \Delta t}{K_{d} / H_{B}+D_{d} / H_{B}}
$$

where $\boldsymbol{K}_{\boldsymbol{d}}$ and $\boldsymbol{D}_{\boldsymbol{d}}$ are the stiffness and damping coefficients, respectively, and $\boldsymbol{F}_{e}$ and $\Delta \boldsymbol{t}$ are the contact force and sampling time, respectively. Figure 8 shows the compliance model. The contact force modulation method makes the robot enter a stable constrained motion zone, after the transition phase.

\section{EXPERIMENTS}

To test a control method and locomotion pattern proposed for a legged robot to carry heavy-loads and move fast on the uneven terrain, a two-legs and two-wheels robot, qRT-2 is designed as shown in Fig. 9. It is a front drive system vehicle with hydraulic linear actuators, motor-powered and is capable of carrying heavy-loads on back legs with a wheel.

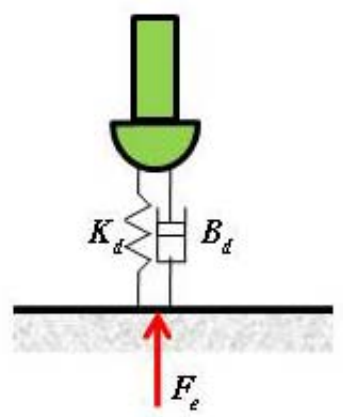

Fig.8 Compliance Model 


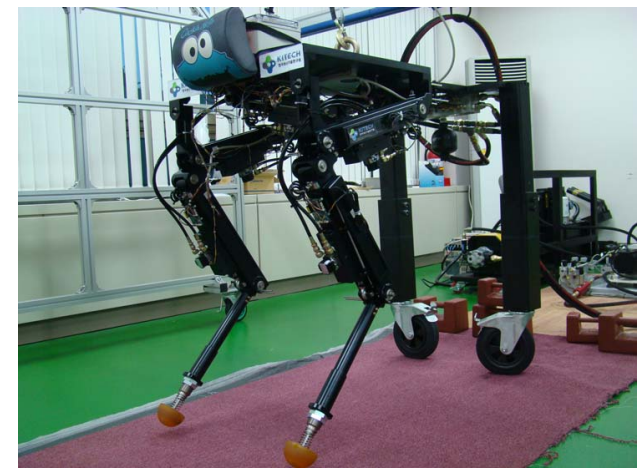

Fig. 9 Configuration of a legged robot, qRT-2

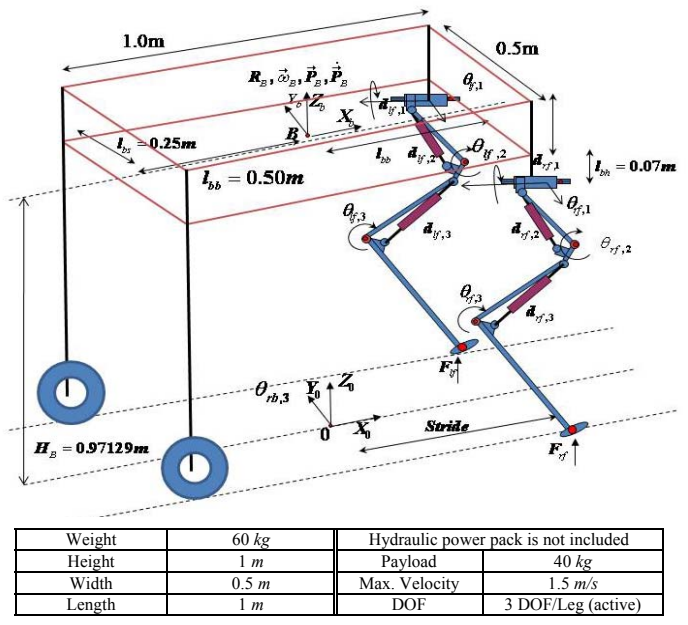

Fig.10 Coordinates and parameters of a legged robot, qRT-2

Fig. 10 shows the detail specification of qRT-2. It is weights about $60 \mathrm{~kg}$ except the hydraulic power pack. It can carry loads of up to $40 \mathrm{~kg}$, amble at over $1.3 \mathrm{~m} / \mathrm{s}$, and climb up and down hills. The overall control architecture for the qRT-2 is composed of the pattern generator, the online compensator, the local controller, and the sensor filtering as shown in Fig. 11. A local controller controls a hydraulic actuator system which consists of a servo value, a DSP board with CAN controller, a hydraulic actuator of the double rod, a LVDT sensor, and a load cell in order to track high level commands from an on-board computer. The LVDT sensors are used to measure the displacement of linear actuators, and an on-board computer is used to control locomotion, servo the legs and handle a wide variety of sensors. Sensors for the locomotion check the joint position and force, the ground contact, the ground load, and the gyroscope. Other sensors check the internal state of qRT-2, monitoring the hydraulic pressure, the oil temperature, the engine temperature, the rpm, the battery charger and the others.

Figure 12(a) shows that qRT-2 using the proposed trajectory pattern generation and control method trots on the even surface. The robot follows the desired trajectory in the error range of less than $0.2 \mathrm{~mm}$. The total flow rate is less than 15.0 lpm because the robot does not turn and namely all the hydraulic actuators which are related to the turning motion do not move. And the supplied hydraulic pressure is about 130 bar. So far, The qRT-2 has trotted at $1.3 \mathrm{~m} / \mathrm{s}$, climbed a 20 degree slope and carried a $40 \mathrm{~kg}$ payload.

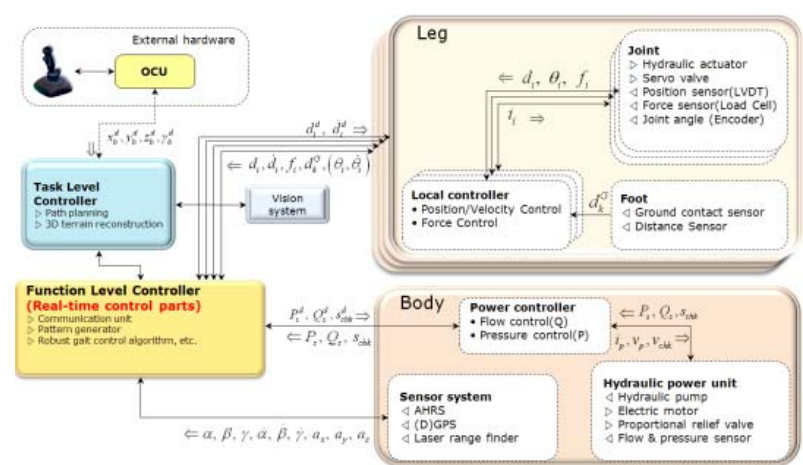

Fig. 11 Control system architecture

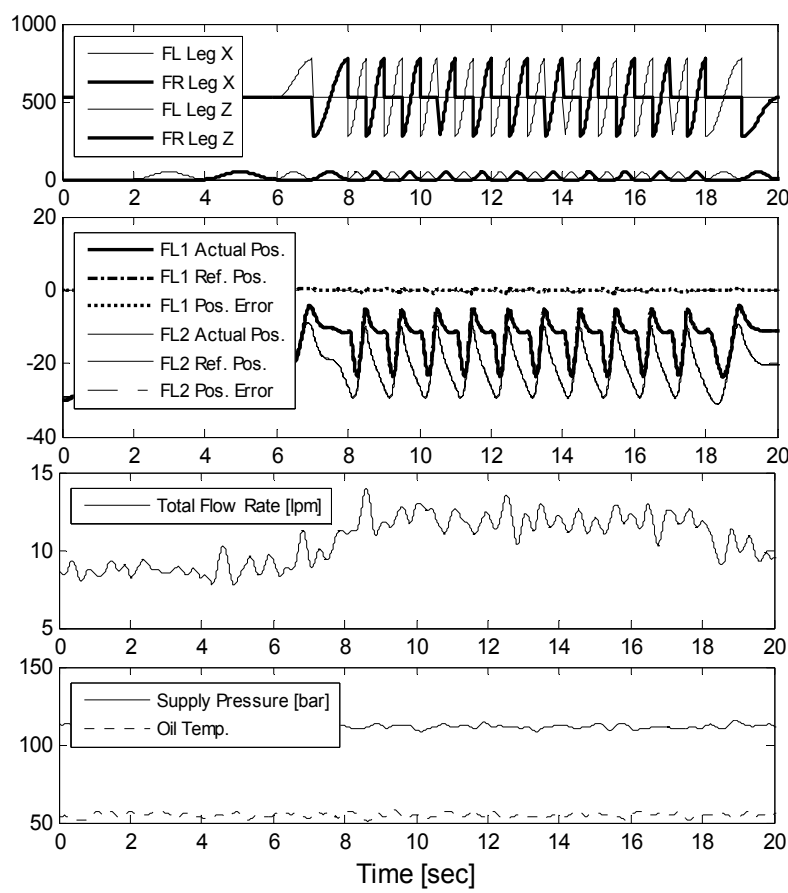

(a) Two upper graphs are related to the foot and joint trajectory to the qRT-2 trots at $0.5 \mathrm{~m} / \mathrm{s}$. And the rest of graphs include the total flow rate, pressure, and oil temperature of the hydraulic power system at the trot gait.

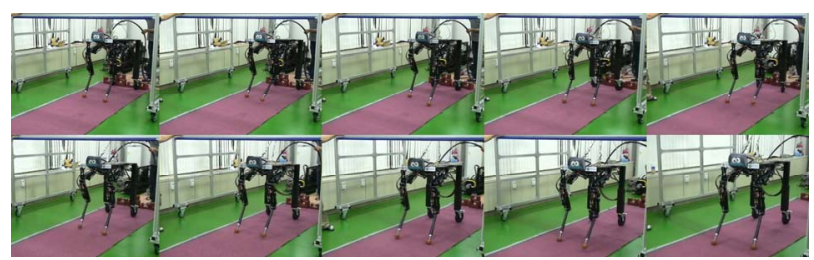

(b) Consecutive scenes of the trots at average velocity of about $0.5 \mathrm{~m} / \mathrm{s}$.

Fig. 12 Experimental results

Figure 12(b) shows the consecutive scenes of trotting of a qRT-2 at average velocity of about $0.5 \mathrm{~m} / \mathrm{s}$. The plot indicates that the proposed trajectory pattern generator and control method make the quadruped robot trot stably. 


\section{CONCLUSION}

This paper was proposed a frequency modulated pattern generator that is easy to change foot trajectories by change of some parameters for real-time system and an online control method for a legged robot to carry heavy-loads and to move fast on the uneven terrain. To test the proposed method, qRT2 which is a front drive system vehicle with the hydraulic linear actuators and can carry heavy-loads on back legs with a wheel is used. It is capable of carrying payloads, operating outdoors, with the static and dynamic mobility, and the fully integrated sensing. It is about $1.0 \mathrm{~m}$ high, $1.0 \mathrm{~m}$ long, $0.5 \mathrm{~m}$ wide, and weights about $60 \mathrm{~kg}$. It has demonstrated the trotting gaits at speeds up to $1.3 \mathrm{~m} / \mathrm{s}$ on the even surface, walked up and down the 20 degree inclines, and walked at $0.7 \mathrm{~m} / \mathrm{s}$ on the uneven surface. Also it has carried over $100 \mathrm{~kg}$ of the total weights including over $40 \mathrm{~kg}$ of the payload. We are currently working to expand the legged robot's rough terrain mobility through the creation of robust locomotion strategies and terrain sensing capabilities.

\section{REFERENCES}

[1] R. Kurazume, S. Hirose, and K. Yoneda, "Feedforward and Feedback Dynamic Trot Gait Control for a Quadruped Walking Vehicle," IEEE Int. Conf. on Robotics and Automation, pp. 3172-3179, 2001

[2] R. Playter, M. Buehler, and M. Raiber, “BigDog," Proc. Of SPIE, Vol. 6230, 2006.

[3] M. Guihard, P. Gorce, and J. G. Fontaine, "SAPPHYR: Legs to pull a wheel structure," IEEE Int. Conf. on Systems, Man and Cybernetics, pp 1303-1308, 1995.

[4] L. R. Palmer II, and D. E. Orin, "3D Control of a High-Speed Quadruped Trot," Industrial Robot: An International Journal, Vol. 33, No. 4, pp. 298-302, 2006.

[5] M. H. Raibert, "Trotting, Pacing, and Bounding by a Quadruped Robot," Journal of Biomechanics, Vol. 23, No. 1, pp. 79-98, 1990.

[6] S. Peng, "A Biologically Inspired Four Legged Walking Robot," Doctoral thesis, Murdoch University, 2006.

[7] H. Tsukagoshi, S. Hirose, and K. Yoneda, "Maneuvering Operations of the Quadruped Walking Robot on the Slope," IEEE Int. Conf. on Intelligent Robots and Systems, pp. 863-869, 1996.

[8] Y. Fukuoka, H. Kimura, and A. H. Cohen, "Adaptive Dynamic Walking of a Quadruped Robot on Irregular Terrain Based on Biological Concepts," The International Journal of Robotics Research, Vol. 22, No.3-4, pp. 187-202, 2003.

[9] C. Ridderstrom, "Legged Locomotion: Balance, Control and Toolsfrom Equation to Action," Doctoral thesis, Royal Institute of Technology, 2003.

[10] J. A. Smith, "Galloping, Bounding and Wheeled-Leg Modes of Locomotion on Underactuated Quadrupedal Robots," Doctoral thesis, McGill University, 2006.

[11] M. H. Raibert, M. Chepponis, and H. B. Brown, JR., "Running on Four Legs As Though They Were One," IEEE Journal of Robotics and Automation, Vol. 2, RA-2, NO. 2, pp. 70-82, 1986. 